\title{
Influence of factor XII deficiency on activated partial thromboplastin time (aPTT) in critically ill patients
}

\author{
Mirjam Bachler ${ }^{1}$ (1) Christian Niederwanger ${ }^{2} \cdot$ Tobias Hell $^{3} \cdot$ Judith Höfer ${ }^{4} \cdot$ Dominic Gerstmeyr $^{5} \cdot$ Bettina Schenk $^{5}$. \\ Benedikt Treml ${ }^{5}$. Dietmar Fries ${ }^{5}$
}

Published online: 23 May 2019

(c) The Author(s) 2019

\begin{abstract}
FXII deficiency results in spontaneous prolongation of activated partial thromboplastin time (aPTT), which is widely used to monitor thromboprophylaxis. Misinterpretation of spontaneously prolonged aPTT may result in omission of thromboembolic treatment or even unnecessary transfusion of blood products. This retrospective analysis was performed to calculate a threshold level of FXII resulting in aPTT prolongation. 79 critically ill patients with spontaneous prolongation of aPTT were included. A correlation analysis and a ROC curve for aPTT prolongation predicted by FXII level were created to find the FXII threshold level. Prolongation of aPTT was associated with disease severity. A significant inverse proportionality between FXII and aPTT was seen. A ROC curve for aPTT prolongation, predicted by FXII level (AUC 0.85; CI 0.76-0.93), revealed a FXII threshold level of $42.5 \%$. Of our patients $50.6 \%$ experienced a FXII deficiency, in $80.0 \%$ of whom we found aPTT to be prolonged without a significantly higher bleeding rate. The FXII deficiency was more common in patients with higher SAPS3 scores, septic shock, transfusion of red blood cells and platelet concentrates as well as in patients receiving renal replacement therapy. Patients with a FXII deficiency and prolonged aPTT less often received anticoagulatory therapy although they were more severely ill. The rate of thromboembolic events was higher in these patients although the difference was not statistically significant. Of all patients with spontaneous aPTT prolongation $50.6 \%$ had a FXII level of $42.5 \%$ or less. Those patients received insufficient thromboembolic prophylaxis.
\end{abstract}

Keywords Anticoagulation · FXII deficiency $\cdot$ aPTT $\cdot$ Critically ill patients $\cdot$ Thromboprophylaxis

Christian Niederwanger

christian.niederwanger@tirol-kliniken.at

1 Institute for Sports Medicine, Alpine Medicine and Health Tourism, UMIT - University for Health Sciences, Medical Informatics and Technology, Eduard Wallnöfer Zentrum 1, 6060 Hall in Tirol, Austria

2 Department of Pediatrics, Pediatric Intensive Care Unit, Pediatrics I, Medical University of Innsbruck, Anichstrasse 35, 6020 Innsbruck, Austria

3 Department of Mathematics, Faculty of Mathematics, Computer Science and Physics, University of Innsbruck, Technikerstraße 13, 6020 Innsbruck, Austria

4 Department of Anesthesiology and Intensive Care Medicine, AUVA Trauma Centre Salzburg, Academic Teaching Hospital of the Paracelsus Medical University, Dr. Franz Rehrl Platz 5, 5020 Salzburg, Austria

5 Department of General and Surgical Critical Care Medicine, Medical University Innsbruck, Anichstrasse 35, 6020 Innsbruck, Austria

\author{
Abbreviations \\ aPTT Activated partial thromboplastin time \\ CRP C-reactive protein \\ DIC Disseminated Intravascular Coagulation \\ FFP Fresh frozen plasma \\ FXII Coagulation factor XII \\ ICU Intensive care unit \\ INR International normalized ratio \\ PC Platelet concentrate \\ PT Prothrombin time \\ RBC Red blood cells \\ RRT Renal replacement therapy \\ SAPS3 Simplified Acute Physiology Score 3 \\ SOFA Sequential Organ Failure Assessment
}




\section{Highlights}

The correct setting of anticoagulation especially in critically ill patients is of existential importance. It is mostly based on the aPTT value. Other parameters, such as factor XII, can corrupt this value.

- FXII deficiency is a common finding among critically ill patients with sepsis.

- FXII levels lower than $42.5 \%$ frequently leads to an apparent prolongation of aPTT.

- aPTT prolongation due to FXII deficiency may result in omission of thromboprophylaxis.

- With such a complex system as the coagulation can aPTT can not be interpreted without reservation for itself. The search for more independent parameters must be further accelerated.

\section{Introduction}

Pathological standard coagulation parameters are frequently observed in critically ill patients despite the absence of any relevant clinical bleeding symptoms. In addition to increased PT and INR levels and decreased platelet numbers, prolongation of the activated partial thromboplastin time (aPTT) is frequently noticed, especially in this population. In general, increased aPTT is sensitive to decreased levels of Factor VIII, IX, XI and XII as well as to the intake of anticoagulants, anti-phospholipid antibodies and von Willebrand disease [1-3]. Since aPTT measurement is often misused as a predictor for bleeding in critically ill patients [4], the detection of prolongation may lead to insufficient or no antithrombotic therapy or even result in the indiscriminate use of coagulation factors or blood products, especially fresh frozen plasma (FFP), or may even cause urgently required surgical interventions to be postponed $[5,6]$.

If the aPTT prolongation does not reflect a hypocoagulant state, but is the consequence of confounding factors in the laboratory assay, corrective actions are problematic. Lupus anticoagulants or contact pathway factor deficiencies, such as prekallikrein or FXII deficiency, without any increased risk for bleeding [6-8] may influence the aPTT result.

In critically ill patients with the need for anticoagulant treatment, prolongation of aPTT without any real clinical bleeding tendency is a major problem since aPTT is widely used to monitor the efficacy of anticoagulation [9-11]. Prolongation of aPTT may lead to inadequate thromboprophylactic drug dosing, or in the worst case to no anticoagulation therapy at all [12] and to exposure to allogenic fresh frozen plasma (FFP) transfusion [13]. FFP transfusions and other countermeasures [14] may cause harm if not indicated accurately $[15,16]$.

Especially in critically ill patients the overall incidence of thromboembolic events (TE) is about 32\% [17]. If antithrombotic therapy is withheld, the rate of deep vein thrombosis (DVT) can increase to up to $60 \%$ in trauma patients and even to $80 \%$ if an acute spinal cord injury is involved [18]. Therefore, correct interpretation of the aPTT is crucial, especially in the critically ill patient population.

aPTT prolongation with the absence of any clinically relevant bleeding symptoms is a common finding in intensive care units. Correct interpretation is difficult, the consequence of which should not be inadequate activities [19]. Even though aPTT prolongation cannot be ascribed solely to FXII deficiency, we chose this factor because of its use as a surrogate marker for contact pathway activation.

Although it has long been known that critically ill patients have increased contact activation with concomitant FXII consumption leading to aPTT prolongation, hardly any studies have dealt with this subject to date.

Since in critically ill patients based on the aPTT time, the current coagulation situation could not be interpreted correctly and thus a wrong treatment decision regarding anticoagulation could be made, we would like to point out in this study that aPTT could be incorrectly prolonged by influencing factors such as FXII deficiency. Therefore, the purpose of this study was to investigate the impact of FXII deficiency on a prolongation of aPTT and thus increase the awareness of treating physicians about the interpretation of aPTT.

\section{Methods}

Primary endpoint of this study was to evaluate whether the FXII levels in patients with and without prolongation of the targeted aPTT range differ and also, as secondary endpoint, to determine a FXII threshold level where aPTT becomes prolonged by a FXII deficiency.

\section{Inclusion of patients}

This retrospective analysis includes clinical data and routine laboratory parameters of 79 critically ill post-surgical patients as well as trauma patients at the General and Surgical Intensive Care Unit of Innsbruck Medical University Hospital.

All medical files of patients with available FXII measurement for whatever reason (e.g. participants in a clinical trial with measurement of various coagulation factors) between 2012 and 2015 were reviewed. A total of 94 patients were found, ten of whom had to be excluded from analysis since 
they had confounding influences on the coagulation Factor XII assay, such as Lupus anticoagulants, or the administration of direct thrombin inhibitor. Five patients in whom it was unclear whether aPTT was prolonged or not, were excluded. A total of 79 patients were suitable for analysis. For five of these 79 patients limited information was available.

The study protocol was approved by the institutional review board of the Medical University of Innsbruck (EKNo. $1151 / 2017$ ).

\section{Data collection}

We collected demographic variables such as age, sex, BMI and the reason for ICU admission. Septic shock was defined as the need for catecholamines. As ICU interventions, extracorporeal circuit treatments and anticoagulation therapy were recorded. aPTT prolongation was defined as assay results above the upper norm value of more than $37 \mathrm{~s}$ in patients not receiving any anticoagulatory medication. A target aPTT of $60 \mathrm{~s}$ was standard in heparin therapy. An overshoot of the targeted upper aPTT range (>60 s) was defined as aPTT prolongation. If a patient had more than one FXII measurement during the entire ICU stay, the time point of the lowest FXII level was chosen for analysis. At such time other available routine parameters such as fibrinogen, platelets, antithrombin, PT, INR, hemoglobin, hematocrit, leukocytes, C-reactive protein, procalcitonin were collected. The number of transfusions received before FXII measurement was recorded and analysed as the total number of transfusion units and only from 3 days before in order to evaluate the short-term effect of transfusion on the FXII levels. We looked out for any diagnosed thromboembolic event and bleeding complication during the ICU stay.

\section{Statistical analysis}

A mathematician (TH) not involved in data acquisition performed the statistical analyses using $\mathrm{R}$, version 3.4.1. All statistical assessments were two-sided and a significance level of $5 \%$ was used. We investigate the relationship between FXII and aPTT using regression analysis and perform ROC curve analysis for an aPTT prolongation predicted by FXII levels; we provide an optimal threshold for FXII as the value corresponding to the point closest to the top-left on the ROC curve. Because the hypothesis of normal distribution was not reasonable for most continuous variables (Shapiro-Wilk normality test) and sample size was not accordingly high, we applied the Wilcoxon rank sum test and Fisher's exact test to assess differences between patients with and without prolonged aPTT as well as between patients with FXII levels above and those with FXII levels below the threshold. We present continuous data as median (IQR) and categorical variables as frequencies (\%) and show effect size and precision with estimated differences between groups for continuous data and odds ratios (OR) for binary variables, with $95 \%$ CIs.

\section{Results}

\section{Patient characteristics}

Eligible for final analysis were 79 patients; median age was 64 years, $26.6 \%$ of the patients were female. The main affected organ system, responsible for ICU admission, was the cardiovascular system (39.2\%) followed by septic complications (17.7\%) as seen in Table 1.

Prolongation of aPTT was observed in $51.9 \%$ of our patient population. Patients with aPTT prolongation had statistically higher SAPS3 $(p<0.0001)$ and SOFA $(p=0.0006)$ scores, namely 78 and 14 patients as compared to 59.5 and ten patients, respectively.

Proven pathogens were found in $74.7 \%$ of our patients, with gram- bacteria being the most common (55.7\%). In the course of ICU stay, on the day of the lowest FXII activity, almost half (44.3\%) of our study patients developed renal failure and needed renal replacement therapy (RRT). Patients' baseline characteristics stratified for aPTT are presented in Table 1.

In Fig. 1, the fitted cubic smoothing spline (black line) reveals a highly nonlinear dependence between FXII and aPTT levels. Inverse proportionality of FXII and aPTT measurement (blue solid line with $95 \%$ CIs as dashed lines) was confirmed by regression analysis $(\mathrm{p}<0.0001)$.

The optimal threshold for FXII levels was calculated at $42.5 \%$. Below this, the FXII deficiency is most likely to confound the aPTT assay, resulting in prolongation of aPTT (Fig. 2).

The optimal threshold for FXII is $42.5 \%$ and corresponds to the point closest to the top-left marked with an asterisk, where a specificity of 0.79 and a sensitivity of 0.78 are achieved.

Of our patients $50.6 \%$ experienced such a FXII deficiency, $80.0 \%$ of whom showed prolongation of aPTT. Disease severity and different treatments were associated with a FXII deficiency below the threshold level of $42.5 \%$ as presented in Table 2.

Patients with FXII deficiency were in general sicker than patients without, as evaluated by SAPS3 scores. FXII deficiency was significantly more common in patients with septic shock. Renal replacement therapy was more often necessary as well as the administration of red blood cell and platelet concentrates until three days before FXII measurement. The coagulation tests are deflected; coagulation factors and platelets are decreased in the deficiency 
Table 1 Characteristics of patients stratified for aPTT not prolonged and aPTT prolonged

\begin{tabular}{|c|c|c|c|c|c|}
\hline Characteristic $^{\mathrm{a}}$ & Total $(n=79)$ & aPTT not prolonged $(=38)$ & aPTT prolonged $(n=41)$ & Estimate with $95 \% \mathrm{CI}^{\mathrm{b}}$ & $\mathrm{p}$ value ${ }^{\mathrm{c}}$ \\
\hline Female gender & $21 / 79(26.6)$ & $8 / 38(21.1)$ & $13 / 41(31.7)$ & $1.73(0.56-5.6)$ & 0.3182 \\
\hline Age (years) & $64(54-73)$ & $58.5(48.25-69.5)$ & $68(59-76)$ & $-8(-14$ to -1$)$ & 0.0197 \\
\hline BMI $\left(\mathrm{kg} / \mathrm{m}^{2}\right)$ & $26.55(22.87-28.7)$ & $26.81(24.78-28.8)$ & $25.6(21.18-28.4)$ & $1.9(-0.5$ to 4.5$)$ & 0.115 \\
\hline \multicolumn{6}{|l|}{ Reason for ICU admission ${ }^{\mathrm{d}}$} \\
\hline Trauma & $8 / 79(10.1)$ & $6 / 38(15.8)$ & $2 / 41(4.9)$ & $0.28(0.03-1.69)$ & 0.1449 \\
\hline Sepsis & $14 / 79(17.7)$ & $2 / 38(5.3)$ & $12 / 41(29.3)$ & $7.28(1.45-71.97)$ & 0.007 \\
\hline MODS & $8 / 79(10.1)$ & $2 / 38(5.3)$ & 6/41 (14.6) & $3.04(0.5-32.83)$ & 0.266 \\
\hline Cardiovascular system $^{\mathrm{e}}$ & $31 / 79(39.2)$ & $16 / 38(42.1)$ & $15 / 41(36.6)$ & $0.8(0.29-2.16)$ & 0.6508 \\
\hline Respiratory insufficiency & $7 / 79(8.9)$ & $2 / 38(5.3)$ & $5 / 41(12.2)$ & $2.47(0.37-27.57)$ & 0.4338 \\
\hline Renal failure & 9/79 (11.4) & $3 / 38(7.9)$ & 6/41 (14.6) & $1.98(0.39$ to 13.23$)$ & 0.4842 \\
\hline Hepatic dysfunction & $1 / 79(1.3)$ & $0 / 38(0)$ & $1 / 41(2.4)$ & $\operatorname{Inf}(0.02$ to $\operatorname{Inf})$ & 1 \\
\hline Intestinal tract complication & $12 / 79(15.2)$ & $6 / 38(15.8)$ & 6/41 (14.6) & $0.92(0.22-3.81)$ & 1 \\
\hline Thromboembolic event & $5 / 79(6.3)$ & $4 / 38(10.5)$ & $1 / 41(2.4)$ & $0.22(0-2.32)$ & 0.1898 \\
\hline \multicolumn{6}{|l|}{ Scores } \\
\hline SAPS3 (pts) & $71(54.5-80.5)$ & $59.5(47.25-71)$ & $78(72-87)$ & $-20(-29$ to -12$)$ & $<0.0001$ \\
\hline SOFA (pts) & $12(8.5-14)$ & $10(7-12.25)$ & $14(11-16)$ & $-4(-6$ to -2$)$ & 0.0006 \\
\hline \multicolumn{6}{|l|}{ Sepsis ${ }^{\mathrm{d}}$} \\
\hline Proven pathogen & $59 / 79(74.7)$ & $26 / 38(68.4)$ & $33 / 41(80.5)$ & $1.89(0.61-6.19)$ & 0.3012 \\
\hline Gram+ & $40 / 79(50.6)$ & $19 / 38(50)$ & $21 / 41(51.2)$ & $1.05(0.4-2.78)$ & 1 \\
\hline Gram- & $44 / 79(55.7)$ & $19 / 38(50)$ & $25 / 41(61)$ & $1.55(0.58-4.2)$ & 0.3702 \\
\hline Fungi & $38 / 79(48.1)$ & $14 / 38(36.8)$ & $24 / 41(58.5)$ & $2.39(0.89-6.62)$ & 0.0722 \\
\hline Viral & $10 / 79(12.7)$ & $5 / 38(13.2)$ & $5 / 41(12.2)$ & $0.92(0.19-4.38)$ & 1 \\
\hline Septic shock & $26 / 74(35.1)$ & $5 / 35(14.3)$ & $21 / 39(53.8)$ & $6.81(2.03-27.29)$ & 0.0005 \\
\hline \multicolumn{6}{|c|}{ Interventions at lowest FXII level } \\
\hline ECMO & $7 / 79(8.9)$ & $4 / 38(10.5)$ & $3 / 41(7.3)$ & $0.67(0.09-4.3)$ & 0.7053 \\
\hline RRT & $35 / 79(44.3)$ & $10 / 38(26.3)$ & $25 / 41(61)$ & $4.29(1.53-12.82)$ & 0.003 \\
\hline Anticoagulation therapy & $51 / 74(68.9)$ & $34 / 35(97.1)$ & $17 / 39(43.6)$ & $0.02(0-0.17)$ & $<0.0001$ \\
\hline Length of ICU stay (days) & $16(8-26)$ & $13(9.25-24)$ & $17(5-26)$ & $-1(-8$ to 5$)$ & 0.7871 \\
\hline
\end{tabular}

${ }^{\text {a } B i n a r y ~ d a t a ~ a r e ~ p r e s e n t e d ~ a s ~ n o . / t o t a l ~ n o . ~(\%), ~ c o n t i n u o u s ~ d a t a ~ a s ~ m e d i a n s ~(25 t h-75 t h ~ p e r c e n t i l e) ~}$

${ }^{\mathrm{b}}$ Estimated odds ratio for binary and median difference for continuous variables

${ }^{c}$ Differences in groups assessed with Fisher's Exact Test for binary variables and Wilcoxon Rank Sum Test for continuous variables

${ }^{\mathrm{d}}$ Multiple selection possible

${ }^{\mathrm{e}}$ Includes post-surgical care or complications

group. Except for procalcitonin, which was significantly higher in the deficiency group, there were no differences in the inflammatory parameters. The rate of thrombosis and bleeding complications was not significantly different in either group although the number of thromboses was higher in patients with a FXII deficiency.

As depicted in Fig. 3, FXII levels significantly differ in patients with aPTT prolongation as compared to patients without $(\mathrm{p}<0.0001)$, and thus confirmed the calculated FXII threshold level of $42.5 \%$. FXII levels were significantly lower $(p=0.003)$ when undergoing renal replacement therapy or transfusion of blood products (total RBC: $\mathrm{p}=0.0021$ and platelet concentrate until three days before FXII measurement: $\mathrm{p}=0.0018$ ) was necessary.

\section{Discussion}

Our study showed a significant inverse proportionality between FXII and aPTT. In addition, a threshold FXII of $42.5 \%$ could be calculated from this, it comes to a significant extension of aPTT. We would like to point out a careful use and careful interpretation of aPTT for therapeutic decisions.

Correct interpretation of so-called standard laboratory coagulation parameters combined with adequate therapeutic response, especially in the critically ill patient population, may contribute to the reduction in thromboembolic complications as well as to the prevention of bleeding complications. Beside routine measurement of PT/INR and platelet count, adjustment of a patient's coagulation and 


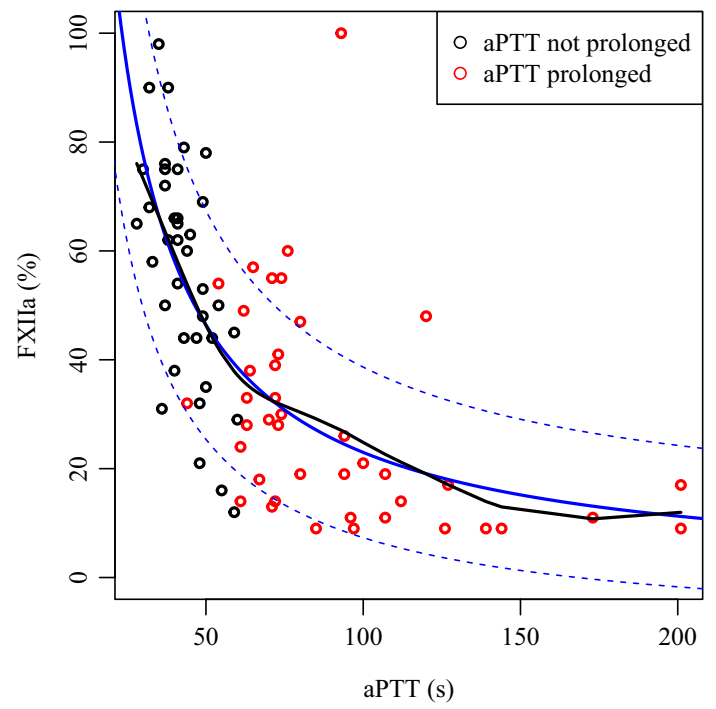

Fig. 1 Inverse proportionality of FXII and aPTT measurement. The black line shows the fitted cubic line. The inverse proportionality is depicted as the blue solid line with $95 \%$ CIs as dashed lines

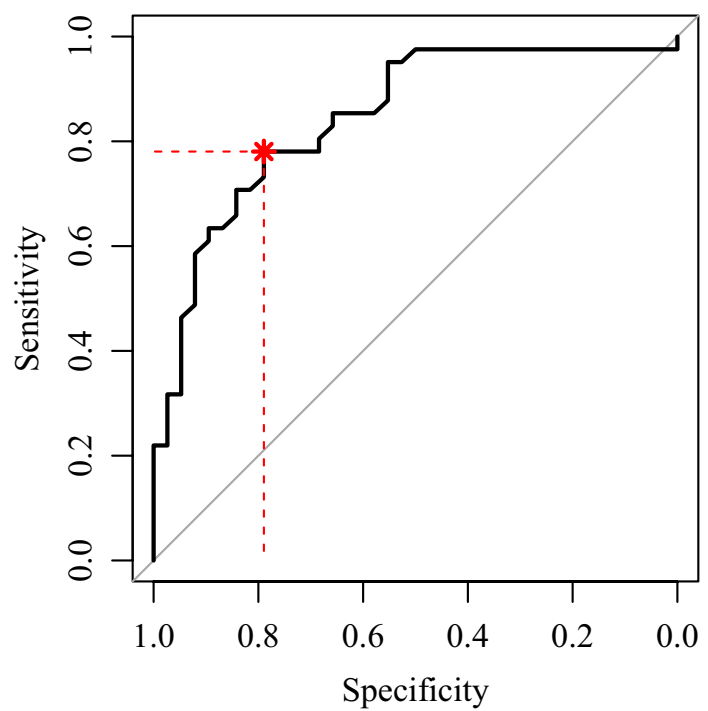

Fig. 2 ROC curve for aPTT prolongation predicted by FXII level: AUC 0.85 (0.76-0.93)

thromboembolic prophylaxis and therapy is usually based on aPTT measurement. In this connection we came to the conclusion that spontaneous prolongation of aPTT measurement in critically ill patients is influenced in nearly $50 \%$ by decreased Factor XII levels. Therefore, spontaneous prolongation of aPTT is neither able to predict bleeding complications nor helpful in predicting thromboembolic complications, whereas a shortened aPTT may identify patients at risk for thromboembolic complications.
Several confounding factors, such as Lupus anticoagulants or FXII deficiency, prolong aPTT without reflecting the patient's bleeding tendency or the efficacy of anticoagulant agents. FXII deficiency can be congenital or acquired and differs among different patient populations [20, 21], but exclusively FXII deficiency was shown to be the most common cause of aPTT prolongation in cancer patients [19]. Only a few studies have investigated the effect of Factor XII deficiency on aPTT and to our knowledge no study has to date been conducted in critically ill patients.

This is the first study reporting about the frequency of FXII deficiency in critically ill patients with spontaneous prolongation of FXII and the influence of FXII deficiency per se on prolongation of aPTT in this patient population. In $50.6 \%$ of our patients with prolonged aPTT, a relevant FXII deficiency was observed. But we did not detect any significant difference in the occurrence of bleeding complications as compared to those patients without FXII deficiency. A FXII threshold level of $42.5 \%$ or less resulted in significant prolongation of aPTT.

In routine practice, spontaneously prolonged aPTT may discourage ICU physicians from initiating thrombosis prophylaxis or therapy. Not only the withholding of antithrombotic drugs, but also the transfusion of fresh frozen plasma in order to normalize the prolonged standard coagulation test is common practice [22, 23].

Our study indicates that FXII deficiency is a common finding among critically ill patients, especially those with sepsis and septic shock. Sepsis severity is associated with elevated procalcitonin levels [24, 25] and, as confirmation thereof, significantly higher procalcitonin levels were also found in our FXII-deficient patients.

As a consequence of infection and inflammation, FXII seems to be consumed by direct activation of FXII by pathogens [26] as well by activation by apoptotic cells via the externalized phosphatidylserines (PS) [27], extracellular nucleic acids such as RNA and DNA [28-30] as happens during neutrophil extracellular trap (NET) formation [31]. Furthermore, activated platelets and the plateletderived microparticles (PMP) activate FXII via the exposed polyphosphates, such as phosphatidylserine (PS) [32]. Patients with septic shock usually receive norepinephrine, which also amplifies the shedding of such procoagulatory PMPs [33].

Patients undergoing continuous renal replacement therapy (RRT) were seen significantly more often to have a FXII deficiency. This could be explained by FXII activation through contact with artificial surfaces of catheters or in the extracorporeal circuits. Once FXII is activated by a surface, up to $60 \%$ of it remains bound to the surface [34], resulting in rapid depletion of FXII in the bloodstream.

FXII deficiency was detected more frequently in patients requiring red blood cell concentrates. Red blood cells are 
Table 2 Patient characteristics shown by FXII above and below threshold of $42.5 \%$

\begin{tabular}{|c|c|c|c|c|c|}
\hline Characteristic $^{\mathrm{a}}$ & Total $(\mathrm{n}=79)$ & FXII $\geq 42.5 \%(=39)$ & FXII $<42.5 \%(n=40)$ & Estimate with $95 \% \mathrm{CI}^{\mathrm{b}}$ & $\mathrm{p}$ value $\mathrm{c}^{\mathrm{c}}$ \\
\hline aPTT prolonged & $41 / 79(51.9)$ & $9 / 39(23.1)$ & $32 / 40(80)$ & $12.77(4.08-45.2)$ & $<0.0001$ \\
\hline Anticoagulation therapy & $51 / 74(68.9)$ & $32 / 36(88.9)$ & $19 / 38(50)$ & $0.13(0.03-0.47)$ & 0.0004 \\
\hline Septic shock & $26 / 74(35.1)$ & $6 / 36(16.7)$ & $20 / 38(52.6)$ & $5.42(1.7-19.75)$ & 0.0015 \\
\hline \multicolumn{6}{|l|}{ Scores } \\
\hline SAPS3 (pts) & $58(25-74.5)$ & $38(15-66)$ & $70(55-82.5)$ & $-28(-44$ to -14$)$ & 0.0002 \\
\hline SOFA (pts) & $8(5-15)$ & $7(4-16)$ & $8(5.75-13.5)$ & $0(-2$ to 2$)$ & 0.705 \\
\hline \multicolumn{6}{|c|}{ Extracorporeal circuit treatments at lowest FXII level } \\
\hline ECMO & $7 / 79(8.9)$ & $3 / 39(7.7)$ & $4 / 40(10)$ & $1.33(0.21-9.72)$ & 1 \\
\hline RRT & $35 / 79(44.3)$ & $10 / 39(25.6)$ & $25 / 40(62.5)$ & $4.73(1.68-14.25)$ & 0.0014 \\
\hline \multicolumn{6}{|c|}{ Total administration of blood products before FXII measurement } \\
\hline Red blood cell concentrate & $56 / 74(75.7)$ & $23 / 36(63.9)$ & $33 / 38(86.8)$ & $3.66(1.05-15.02)$ & 0.0301 \\
\hline FFP & $8 / 74(10.8)$ & $2 / 36(5.6)$ & $6 / 38(15.8)$ & $3.14(0.51-34.03)$ & 0.2627 \\
\hline Platelet concentrate & $25 / 74(33.8)$ & $8 / 36(22.2)$ & $17 / 38(44.7)$ & $2.79(0.93-9.02)$ & 0.0513 \\
\hline \multicolumn{6}{|c|}{ Administration of blood products within 3 days before FXII measurement } \\
\hline Red blood cell concentrate & 25/74 (33.8) & $10 / 36(27.8)$ & $15 / 38(39.5)$ & $1.68(0.58-5.1)$ & 0.3322 \\
\hline FFP & $5 / 74(6.8)$ & $1 / 36(2.8)$ & $4 / 38(10.5)$ & $4.05(0.38-208.23)$ & 0.3585 \\
\hline Platelet concentrate & $15 / 74(20.3)$ & $3 / 36(8.3)$ & $12 / 38(31.6)$ & $4.97(1.17-30.31)$ & 0.0194 \\
\hline \multicolumn{6}{|l|}{ Biological parameters } \\
\hline Hemoglobin $(\mathrm{g} / \mathrm{l})$ & $89(83-94.5)$ & $90(84.5-95.5)$ & $87(79-92.5)$ & $3.54(-1$ to 8$)$ & 0.113 \\
\hline Hematocrit (\%) & $26.5(24.65-28.4)$ & $26.7(24.7-28.45)$ & $26.1(23.95-28.33)$ & 0.7 ( -0.8 to 2.2$)$ & 0.3801 \\
\hline aPTT (s) & $60(42-78)$ & $43(37.5-54)$ & $72.5(61-101.75)$ & $-29(-40$ to -20$)$ & $<0.0001$ \\
\hline PT Quick (\%) & $59(43-77)$ & $75(62-84.5)$ & $45(32-57)$ & $29(21-37)$ & $<0.0001$ \\
\hline INR & $1.4(1.2-1.7)$ & $1.2(1.1-1.3)$ & $1.6(1.4-2.2)$ & $-0.4(-0.7$ to -0.3$)$ & $<0.0001$ \\
\hline Fibrinogen (mg/dl) & $376(228.5-570)$ & $518(362-656)$ & $250(201.5-381.5)$ & $221(127-304)$ & $<0.0001$ \\
\hline Antithrombin (\%) & $51(35-66)$ & $64(56.5-74)$ & $37.5(19-49.25)$ & $27(19-35)$ & $<0.0001$ \\
\hline Platelets (G/l) & $103(69-180)$ & $127(97.5-212.5)$ & $73(45.5-154.75)$ & $52(26-84)$ & 0.0009 \\
\hline Leukocytes (G/l) & $10.6(7.4-13.65)$ & $9.7(7.95-12.7)$ & $11.3(6.88-14.38)$ & $-0.6(-3.1$ to 1.8$)$ & 0.6449 \\
\hline C-reactive protein (mg/dl) & $10.67(5.18-15.25)$ & $10.88(5.21-19.92)$ & $10.37(5.46-14.3)$ & $0.7(-3.63$ to 4.81$)$ & 0.8322 \\
\hline Procalcitonin $(\mu \mathrm{g} / \mathrm{l})$ & $2.46(0.65-5.8)$ & $0.61(0.27-2.02)$ & $4.19(1.93-11.3)$ & $-2.86(-4.76$ to -1.48$)$ & $<0.0001$ \\
\hline Thromboembolic event & $22 / 79(27.8)$ & $7 / 39(17.9)$ & $15 / 40(37.5)$ & $2.71(0.88-9.13)$ & 0.0784 \\
\hline Bleeding complication & $22 / 79(27.8)$ & $8 / 39(20.5)$ & $14 / 40(35)$ & $2.07(0.68-6.65)$ & 0.2101 \\
\hline
\end{tabular}

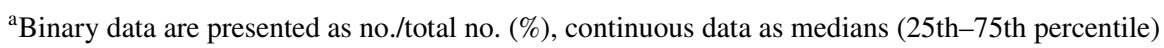

${ }^{\mathrm{b}}$ Estimated odds ratio for binary and median difference for continuous variables

${ }^{c}$ Differences in groups assessed with Fisher's exact test for binary variables and the Wilcoxon rank sum test for continuous variables

known to shed procoagulant microparticles [35]. However, this finding might also be attributed to the severity of the underlying disease and the related need for transfusion. Nevertheless, not only red blood cell concentrates, but also platelet transfusion may affect consumption of FXII. The significantly lower FXII levels in patients receiving platelet concentrates within three days before measurement indicate that the administration of platelet concentrates may lead to a FXII deficiency. This might happen via activated platelets derived from concentrates, which can expose polyphosphates [32] and subsequently activate FXII resulting in a deficiency. This is emphasized by the fact that platelet activation markers in platelet concentrates increase with storage time [36, 37] and also contain platelet-derived particles [36-38], both of which can activate FXII.

A previous study indicated that low or persistently low serial Factor XII values might be associated with poor prognosis [39]. The reason for this could be the preceding activation of the contact pathway, which might lead to an elevated thrombotic risk and inflammation reaction. Early inhibition of FXII could be a promising anti-thrombotic and anti-inflammatory treatment strategy, especially in sepsis. Furthermore, blocking FXII may also be a suitable alternative for anticoagulation of extracorporeal devices like RRT and ECMO by inhibiting contact activation without increasing the bleeding risk [40-42]. 

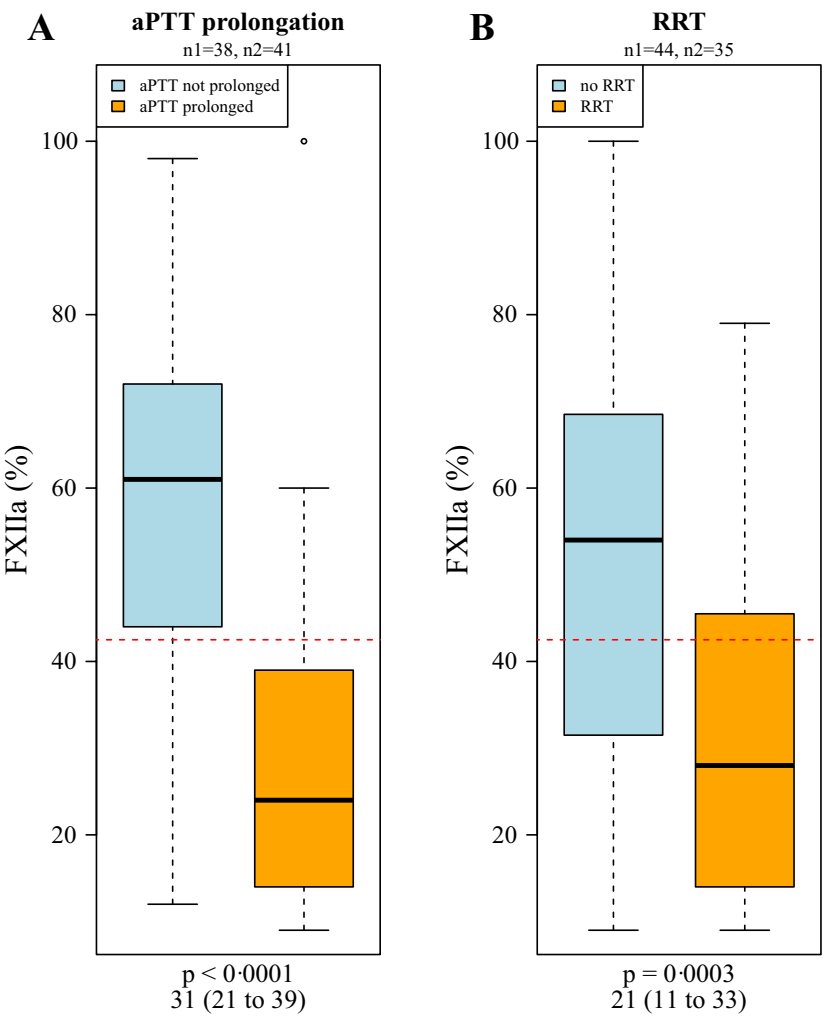

Fig. 3 FXII levels depending on aPTT prolongation, RRT and transfusion. FXII levels differ in dependence on aPTT prolongation (a) and significant treatments (renal replacement therapy $\mathbf{b}$; cumulative

The retrospective design of the study is limiting and precludes assessment of causal relationships. The influences of other factors such as the occurrence of antiphospholipid syndrome, FIX and FXI deficiency or deficiency of plasma kallikrein on the aPTT assay were not assessed and should definitely be considered in a further prospective study. The need to re-calculate the FXII cut-off value for aPTT prolongation after adjustment for deficiencies of these factors is given and the FXII threshold level will probably shift. Furthermore, the presence of disseminated intravascular coagulation (DIC) should also be evaluated, since this can lead to prolonged aPTT. DIC is possible although platelet count and fibrinogen levels in our study are quite high for this disease; at any rate, low FXII levels may indicate DIC [43].

Isolated aPTT prolongation in critically ill patients is rare since aPTT prolongation is most likely due to the sum of pathophysiological processes including liver synthesis.

Nevertheless, awareness about the influence of inhibited FXII on the prolongation of aPTT must be particularly emphasized.
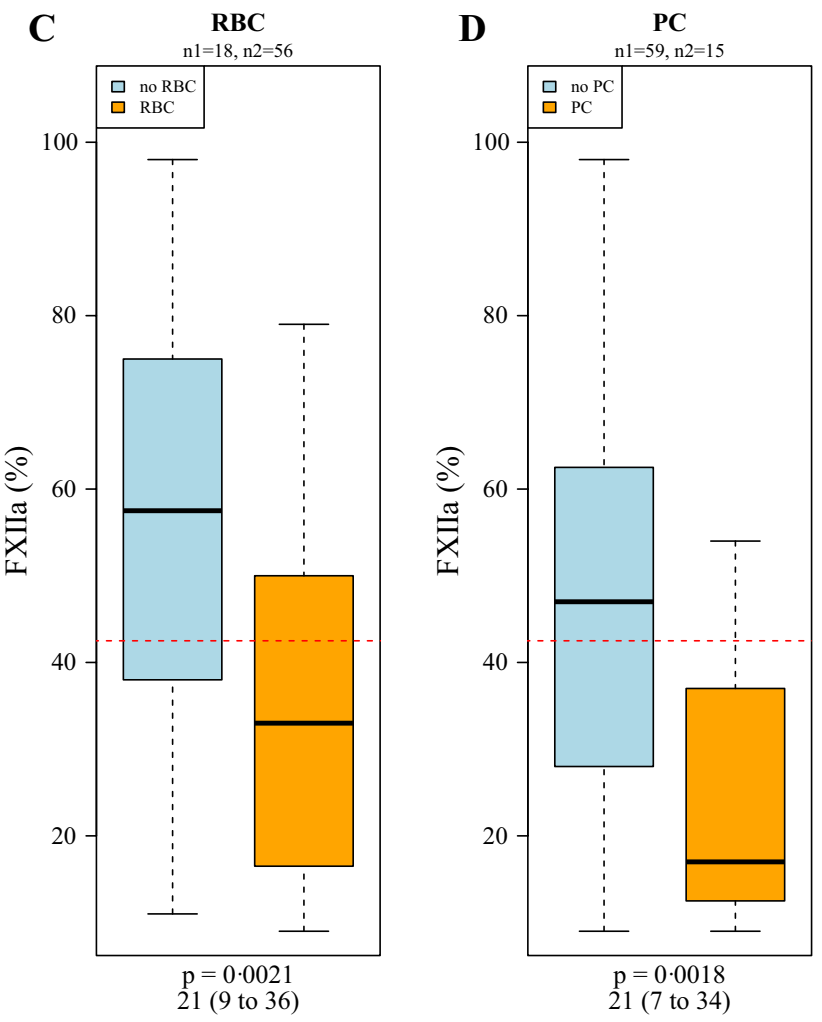

red blood cells $\mathbf{c}$; platelet concentrates d). The red dashed line depicts the calculated $42.5 \%$ threshold level of FXII

\section{Conclusion}

The effect of FXII deficiency is an underestimated problem in critically ill patients. Correct interpretation of prolonged aPTT remains a challenging problem in critical care medicine.

Acknowledgements Open access funding provided by University of Innsbruck and Medical University of Innsbruck.

Funding For this retrospective study funding was received from CSL Behring.

\section{Compliance with ethical standards}

Conflict of interest MB has received funding from CSL Behring $\mathrm{GmbH}$ for the conduction of this retrospective study. She has also received travel grants from LFB Biomedicaments, Baxter GmbH, CSL Behring GmbH, Mitsubishi Tanabe and non-financial support from TEM International outside the submitted work. DF has received study funding, honoraria for consultancy and board activity from Astra Zeneca, AOP orphan, Baxter, Baer, BBraun, Biotest, CSL Behring, Delta Select, Dae Behring, Edwards, Fresenius, Glaxo, Haemoscope, 
Hemogem, Lilly, LFB, Mitsubishi Pharma, NovoNordisk, Octapharm, Pfizer, Tem-Innovation outside the submitted work. All other authors state that they have no competing interests in regard to this study.

Open Access This article is distributed under the terms of the Creative Commons Attribution 4.0 International License (http://creativeco mmons.org/licenses/by/4.0/), which permits unrestricted use, distribution, and reproduction in any medium, provided you give appropriate credit to the original author(s) and the source, provide a link to the Creative Commons license, and indicate if changes were made.

\section{References}

1. Dhainaut JF, Yan SB, Joyce DE, Pettila V, Basson B, Brandt JT, Sundin DP, Levi M (2004) Treatment effects of drotrecogin alfa (activated) in patients with severe sepsis with or without overt disseminated intravascular coagulation. J Thromb Haemost 2(11):1924-1933. https://doi.org/10.111 1/j.1538-7836.2004.00955.x

2. Benediktsson S, Frigyesi A, Kander T (2017) Routine coagulation tests on ICU admission are associated with mortality in sepsis: an observational study. Acta Anaesthesiol Scand 61(7):790-796. https://doi.org/10.1111/aas.12918

3. Tauber H, Innerhofer P, Breitkopf R, Westermann I, Beer R, El Attal R, Strasak A, Mittermayr M (2011) Prevalence and impact of abnormal ROTEM(R) assays in severe blunt trauma: results of the 'Diagnosis and Treatment of Trauma-Induced Coagulopathy (DIA-TRE-TIC) study'. Br J Anaesth 107(3):378-387. https://doi. org/10.1093/bja/aer158

4. Uhl L, Assmann SF, Hamza TH, Harrison RW, Gernsheimer T, Slichter SJ (2017) Laboratory predictors of bleeding and the effect of platelet and RBC transfusions on bleeding outcomes in the PLADO trial. Blood 130(10):1247-1258. https://doi.org/10.1182/ blood-2017-01-757930

5. Tagariello G, Radossi P, Salviato R, Zardo M, De Valentin L, Basso M, Castaman G (2017) Clinical relevance of isolated prolongation of the activated partial thromboplastin time in a cohort of adults undergoing surgical procedures. Blood Transfus 15(6):557-561. https://doi.org/10.2450/2016.0047-16

6. van Veen JJ, Laidlaw S, Swanevelder J, Harvey N, Watson C, Kitchen S, Makris M (2009) Contact factor deficiencies and cardiopulmonary bypass surgery: detection of the defect and monitoring of heparin. Eur J Haematol 82(3):208-212

7. Dietzel H, Lutze G, Katzel R, Liebscher K (2003) Prekallikrein (Fletcher Factor) deficiency and prolongation of APTT reaction. Medizinische Klinik (Munich, Germany: 1983) 98(10):587-590. https://doi.org/10.1007/s00063-003-1299-0

8. Li R, Swaelens C, Vandermijnsbrugge F, Cantinieaux B (2016) Applying a direct aPTT ratio (PlatelinLS/ActinFS) permits to identify rapidly and reliably a bleeding-related factor deficiency or a lupus anticoagulant sequential to an isolated prolongation of aPTT in paediatric pre-operative screening. Eur J Haematol 96(6):578-585. https://doi.org/10.1111/ejh.12634

9. Garcia DA, Baglin TP, Weitz JI, Samama MM (2012) Parenteral anticoagulants: antithrombotic Therapy and Prevention of Thrombosis, 9th ed: American College of Chest Physicians EvidenceBased Clinical Practice Guidelines. Chest 141(2 Suppl):e24Se43S. https://doi.org/10.1378/chest.11-2291

10. Fitousis K, Klasek R, Mason PE, Masud F (2017) Evaluation of a pharmacy managed heparin protocol for extracorporeal membrane oxygenation patients. Perfusion 32(3):238-244. https://doi. org/10.1177/0267659116678057
11. Atallah S, Liebl M, Fitousis K, Bostan F, Masud F (2014) Evaluation of the activated clotting time and activated partial thromboplastin time for the monitoring of heparin in adult extracorporeal membrane oxygenation patients. Perfusion 29(5):456-461

12. van Roessel S, Middeldorp S, Cheung YW, Zwinderman AH, de Pont AC (2014) Accuracy of aPTT monitoring in critically ill patients treated with unfractionated heparin. Neth J Med 72(6):305-310

13. Chowdary P, Saayman AG, Paulus U, Findlay GP, Collins PW (2004) Efficacy of standard dose and $30 \mathrm{ml} / \mathrm{kg}$ fresh frozen plasma in correcting laboratory parameters of haemostasis in critically ill patients. Br J Haematol 125(1):69-73

14. Innerhofer P, Fries D, Mittermayr M, Innerhofer N, von Langen D, Hell T, Gruber G, Schmid S, Friesenecker B, Lorenz IH, Strohle M, Rastner V, Trubsbach S, Raab H, Treml B, Wally D, Treichl B, Mayr A, Kranewitter C, Oswald E (2017) Reversal of trauma-induced coagulopathy usingfirst-line coagulation factor concentrates or fresh frozen plasma (RETIC): a single-centre, parallel-group, open-label, randomised trial. Lancet Haematol 4(6):e258-e271

15. Sarani B, Dunkman WJ, Dean L, Sonnad S, Rohrbach JI, Gracias VH (2008) Transfusion of fresh frozen plasma in critically ill surgical patients is associated with an increased risk of infection. Crit Care Med 36(4):1114-1118. https://doi.org/10.1097/CCM.0b013 e318168f89d

16. Dara SI, Rana R, Afessa B, Moore SB, Gajic O (2005) Fresh frozen plasma transfusion in critically ill medical patients with coagulopathy. Crit Care Med 33(11):2667-2671

17. Schramm D, Bach AG, Meyer HJ, Surov A (2016) Thrombotic events as incidental finding on computed tomography in intensive care unit patients. Thromb Res 141:171-174. https://doi. org/10.1016/j.thromres.2016.03.030

18. Attia J, Ray JG, Cook DJ, Douketis J, Ginsberg JS, Geerts WH (2001) Deep vein thrombosis and its prevention in critically ill adults. Arch Intern Med 161(10):1268-1279

19. Shin DY, Lee HR, Kang HJ, Na II, Chang YH, Yang SH (2015) Prevalent factor XII deficiency in cancer patients with isolated aPTT prolongation. Blood Res 50(2):114-117

20. Halbmayer WM, Haushofer A, Schon R, Mannhalter C, Strohmer E, Baumgarten K, Fischer M (1994) The prevalence of moderate and severe FXII (Hageman factor) deficiency among the normal population: evaluation of the incidence of FXII deficiency among 300 healthy blood donors. Thromb Haemost 71(1):68-72

21. Chng WJ, Sum C, Kuperan P (2005) Causes of isolated prolonged activated partial thromboplastin time in an acute care general hospital. Singapore Med J 46(9):450-456

22. Stanworth SJ, Walsh TS, Prescott RJ, Lee RJ, Watson DM, Wyncoll D (2011) A national study of plasma use in critical care: clinical indications, dose and effect on prothrombin time. Crit Care (London, England) 15(2):5

23. Triulzi D, Gottschall J, Murphy E, Wu Y, Ness P, Kor D, Roubinian N, Fleischmann D, Chowdhury D, Brambilla D (2015) A multicenter study of plasma use in the United States. Transfusion 55(6):1313-1319

24. Anand D, Das S, Ray S, Bhargava S, Srivastava LM (2014) Interrelationship between procalcitonin and organ failure in sepsis. Indian J Clin Biochem 29(1):93-96

25. Jiang L, Feng B, Gao D, Zhang Y (2015) Plasma concentrations of copeptin, C-reactive protein and procalcitonin are positively correlated with APACHE II scores in patients with sepsis. J Int Med Res 43(2):188-195

26. Mattsson E, Herwald H, Cramer H, Persson K, Sjobring U, Bjorck L (2001) Staphylococcus aureus induces release of bradykinin in human plasma. Infect Immun 69(6):3877-3882. https://doi. org/10.1128/iai.69.6.3877-3882.2001 
27. Yang A, Chen F, He C, Zhou J, Lu Y, Dai J, Birge RB, Wu Y (2017) The procoagulant activity of apoptotic cells is mediated by interaction with factor XII. Front Immunol 8:1188. https://doi. org/10.3389/fimmu.2017.01188

28. Kannemeier C, Shibamiya A, Nakazawa F, Trusheim H, Ruppert C, Markart P, Song Y, Tzima E, Kennerknecht E, Niepmann M, von Bruehl ML, Sedding D, Massberg S, Gunther A, Engelmann B, Preissner KT (2007) Extracellular RNA constitutes a natural procoagulant cofactor in blood coagulation. Proc Natl Acad Sci USA 104(15):6388-6393. https://doi.org/10.1073/pnas.06086 47104

29. Gajsiewicz JM, Smith SA, Morrissey JH (2017) Polyphosphate and RNA differentially modulate the contact pathway of blood clotting. J Biol Chem 292(5):1808-1814

30. Pfeiler S, Stark K, Massberg S, Engelmann B (2017) Propagation of thrombosis by neutrophils and extracellular nucleosome networks. Haematologica 102(2):206-213

31. Park HS, Gu J, You HJ, Kim JE, Kim HK (2016) Factor XII-mediated contact activation related to poor prognosis in disseminated intravascular coagulation. Thromb Res 138:103-107

32. Wang Y, Zhang S, Luo L, Norstrom E, Braun OO, Morgelin M, Thorlacius H (2018) Platelet-derived microparticles regulates thrombin generation via phophatidylserine in abdominal sepsis. J Cell Physiol 233(2):1051-1060. https://doi.org/10.1002/jcp.25959

33. Giacomazzi A, Degan M, Calabria S, Meneguzzi A, Minuz P (2016) Antiplatelet agents inhibit the generation of plateletderived microparticles. Front Pharmacol 7:314. https://doi. org/10.3389/fphar.2016.00314

34. Wiggins RC, Bouma BN, Cochrane CG, Griffin JH (1977) Role of high-molecular-weight kininogen in surface-binding and activation of coagulation Factor XI and prekallikrein. Proc Natl Acad Sci USA 74(10):4636-4640

35. Bouchard BA, Orfeo T, Keith HN, Lavoie EM, Gissel M, Fung M, Mann KG (2017) Microparticles formed during storage of red blood cell units support thrombin generation. J Trauma Acute Care Surg. https://doi.org/10.1097/ta.0000000000001759

36. Marcoux G, Duchez AC, Rousseau M, Levesque T, Boudreau LH, Thibault L, Boilard E (2017) Microparticle and mitochondrial release during extended storage of different types of platelet concentrates. Platelets 28(3):272-280. https://doi.org/10.1080/09537 104.2016.1218455
37. Rank A, Nieuwland R, Liebhardt S, Iberer M, Grutzner S, Toth B, Pihusch R (2011) Apheresis platelet concentrates contain plateletderived and endothelial cell-derived microparticles. Vox Sang 100(2):179-186. https://doi.org/10.1111/j.1423-0410.2010.01385 .x

38. Maurer-Spurej E, Larsen R, Labrie A, Heaton A, Chipperfield $\mathrm{K}$ (2016) Microparticle content of platelet concentrates is predicted by donor microparticles and is altered by production methods and stress. Transfus Apheresis Sci 55(1):35-43. https://doi. org/10.1016/j.transci.2016.07.010

39. Pixley RA, Zellis S, Bankes P, DeLa Cadena RA, Page JD, Scott CF, Kappelmayer J, Wyshock EG, Kelly JJ, Colman RW (1995) Prognostic value of assessing contact system activation and factor $\mathrm{V}$ in systemic inflammatory response syndrome. Crit Care Med 23(1):41-51

40. Kokoye Y, Ivanov I, Cheng Q, Matafonov A, Dickeson SK, Mason S, Sexton DJ, Renne T, McCrae K, Feener EP, Gailani D (2016) A comparison of the effects of factor XII deficiency and prekallikrein deficiency on thrombus formation. Thromb Res 140:118-124

41. Nickel KF, Labberton L, Long AT, Langer F, Fuchs TA, Stavrou EX, Butler LM, Renne T (2016) The polyphosphate/factor XII pathway in cancer-associated thrombosis: novel perspectives for safe anticoagulation in patients with malignancies. Thromb Res 141(2):30353-X

42. Hopp S, Nolte MW, Stetter C, Kleinschnitz C, Siren AL, AlbertWeissenberger C (2017) Alleviation of secondary brain injury, posttraumatic inflammation, and brain edema formation by inhibition of factor XIIa. J Neuroinflamm 14(1):017-0815

43. Akaike H (1974) A new look at the statistical model identification. IEEE Trans Autom Control 19(6):716-723. https://doi. org/10.1109/tac.1974.1100705

Publisher's Note Springer Nature remains neutral with regard to jurisdictional claims in published maps and institutional affiliations. 